$R M x A C, \mathbf{5 3}, 127-133(2021)$

(c) 2021: Instituto de Astronomía, Universidad Nacional Autónoma de México

https://doi.org/10.22201/ia.14052059p.2021.53.25

\title{
ROLE OF THE 3.6M DOT TO INVESTIGATE CONNECTIONS BETWEEN LONG-GRBS AND CORE-COLLAPSE SNE
}

\author{
Amit Kumar ${ }^{1,2}$, Shashi B. Pandey ${ }^{1}$, Rahul Gupta ${ }^{1,3}$, Amar Aryan ${ }^{1,3}$, Alberto J. Castro-Tirado ${ }^{4,5}$, and \\ Nameeta Brahme ${ }^{2}$
}

\section{RESUMEN}

El telescopio DOT de 3.6m recientemente instalado en Nainital (Uttarakhand) es una instalación novedosa para la astronomía. Debido a la ubicación de la India en cuanto a longitud terrestre, podría usarse para estudiar nuevos fenómenos transitorios reportados por una red global de telescopios robóticos. Las observaciones resultantes de las imágenes realizadas por la CCD $4 \mathrm{~K} \times 4 \mathrm{~K}$ en el DOT de $3.6 \mathrm{~m}$ serán muy útiles en el futuro cercano para comprender los diferentes aspectos físicos de los eventos críticos en el tiempo, por ejemplo, estallidos de rayos gamma (GRB), supernovas, objetos emisores de ondas gravitatorias, etc. Usando la CCD en modo imagen con filtros de banda ancha (Bessel $U B V R I$ y SDSS ugriz) subtendiendo $\sim 6.5^{\prime} \times 6.5^{\prime}$, se pueden obtener imágenes para intentar varios objetivos científicos en sinergia con otras observaciones multibanda. En este estudio, presentamos un análisis de datos en banda R no publicados de GRB 171205A / SN 2017iuk que abarcan entre 12 y 105 días desde el estallido, que se observó utilizando el DOT de 3,6 m con la CCD de $4 \mathrm{~K} \times 4 \mathrm{~K}$ en modo imagen. En la curva de luz en banda $\mathrm{R}$, un aumento de flujo parece comenzar desde aproximadamente 3 días, presentando un pico de emisión aproximadamente a los 15 días después del estallido, lo que representa una clara evidencia fotométrica de la asociación de una SN con GRB 171205A.

\section{ABSTRACT}

Newly installed 3.6m DOT at Nainital (Uttarakhand) is a novel facility for the time domain astronomy. Because of the longitudinal advantage of India, it could be used to study new transients reported by a global network of robotic telescopes. Observations with the $4 \mathrm{~K} \times 4 \mathrm{~K}$ CCD Imager at the axial port of the $3.6 \mathrm{~m}$ DOT will be very helpful in the near future towards understanding the different physical aspects of time-critical events, e.g., Gamma-ray bursts (GRBs), Supernovae, Gravitational wave candidates, etc. Using the Imager with broadband filters (Bessel UBVRI and SDSS ugriz), $\sim 6.5^{\prime} \times 6.5^{\prime}$ images could be obtained to attempt various science goals in synergy with other multi-band facilities. In this study, we present an analysis of unpublished $R$-band data of GRB 171205A/SN 2017iuk spanning between $\sim 12$ to 105 days since burst, that observed using the $3.6 \mathrm{~m}$ DOT with $4 \mathrm{~K} \times 4 \mathrm{~K}$ CCD Imager. In the $R$-band light curve, a bump appears to start from $\sim 3$ days, which shows the peak at $\sim 15$ days after the burst, clearly indicates photometric evidence of association of SN with GRB 171205A.

Key Words: gamma ray burst: general — instrumentation: miscellaneous — supernovae: general — telescopes

\section{INTRODUCTION}

The longitudinal advantage of Aryabhatta Research Institute of Observational Sciences (ARIES), Nainital, India (Devasthal workstation: Latitude: $29^{\circ} 23^{\prime}$ North; Longitude: $79^{\circ} 41^{\prime}$ East; Altitude: $2540 \mathrm{~m}$ ) is very favorable for the observations of

\footnotetext{
${ }^{1}$ Aryabhatta Research Institute of Observational Sciences, Manora Peak, Nainital, 263 002, India.

${ }^{2}$ School of Studies in Physics and Astrophysics, Pandit Ravishankar Shukla University, Chattisgarh, 492 010, India.

${ }^{3}$ Department of Physics, Deen Dayal Upadhyaya Gorakhpur University, Gorakhpur, 273009, India.

${ }^{4}$ Instituto de Astrofísica de Andalucía (IAA-CSIC), Glorieta de la Astronomía s/n, E-18008, Granada, Spain.

${ }^{5}$ Unidad Asociada Departamento de Ingeniería de Sistemas y Automática, E.T.S. de Ingenieros Industriales, Universidad de Málaga, E-29071, Spain.
}

time-critical events, e.g., afterglows of gamma-ray bursts (GRBs, Kumar 2015). Since 1999, the 1.04m Sampurnanand Telescope (ST) and 1.3m Devasthal Fast Optical Telescope (DFOT) at ARIES, Nainital are also contributing to research regarding the observations of the optical afterglow of GRBs (Sagar 2000). Now, recently commissioned new technology $3.6 \mathrm{~m}$ Devasthal Optical Telescope (DOT) is very useful to understand the physical mechanism, environment, and the possible progenitors of energetic timecritical events like GRBs, SNe, etc. For deep optical imaging, the $4 \mathrm{~K} \times 4 \mathrm{~K}$ CCD Imager as a first light instrument with $15 \mu \mathrm{m}$ pixel size mounted on the axial port of the 3.6m DOT is used (Pandey et al. 2018). The Imager is capable of covering various science goals within field of view of $\approx 6.5^{\prime} \times 6.5^{\prime}$. Apart from 
TABLE 1

PROPERTIES OF THE WELL OBSERVED LGRBS-SNE

\begin{tabular}{|c|c|c|c|c|c|c|}
\hline L-GRB & CCSN & $\begin{array}{l}\text { Redshift } \\
(z)\end{array}$ & $\begin{array}{c}T_{90} \\
\text { (seconds) }\end{array}$ & $\begin{array}{c}E_{\gamma, \text { iso }} \\
\left(10^{52} \text { erg }\right)\end{array}$ & $\begin{array}{c}E_{p} \\
(\mathrm{keV})\end{array}$ & $\begin{array}{c}L_{\text {iso }} \\
\left(\operatorname{erg~s}^{-1}\right)\end{array}$ \\
\hline 980425 & 1998bw & 0.00867 & 18 & $0.000086(0.000002)$ & $55(21)$ & $4.80 \times 10^{46}$ \\
\hline 011121 & $2001 \mathrm{ke}$ & 0.362 & 47 & $7.8(2.1)$ & $793(265)$ & $2.26 \times 10^{51}$ \\
\hline 021211 & $20021 \mathrm{t}$ & 1.004 & 2.8 & $1.12(0.13)$ & $127(52)$ & $8.02 \times 10^{51}$ \\
\hline 030329 & $2003 \mathrm{dh}$ & 0.16867 & 22.76 & $1.5(0.3)$ & $100(23)$ & $7.70 \times 10^{50}$ \\
\hline 031203 & $2003 \mathrm{lw}$ & 0.10536 & 37 & $0.0086(0.004)$ & $<200$ & $2.55 \times 10^{48}$ \\
\hline 050525A & $2005 \mathrm{nc}$ & 0.606 & 8.84 & $2.5(0.43)$ & $127(10)$ & $4.54 \times 10^{51}$ \\
\hline 060218 & 2006aj & 0.03342 & 2100 & $0.0053(0.0003)$ & $4.9(0.3)$ & $2.60 \times 10^{46}$ \\
\hline 081007 & $2008 \mathrm{hw}$ & 0.5295 & 9.01 & $0.15(0.04)$ & $61(15)$ & $2.54 \times 10^{50}$ \\
\hline 091127 & $2009 \mathrm{nz}$ & 0.49044 & 7.42 & $1.5(0.2)$ & $35.5(1.5)$ & $3.01 \times 10^{51}$ \\
\hline 100316D & $2010 \mathrm{bh}$ & 0.0592 & 1300 & $>0.0059$ & $26(16)$ & $4.80 \times 10^{46}$ \\
\hline 101219B & 2010ma & 0.55185 & 51 & $0.42(0.05)$ & $70(8)$ & $1.27 \times 10^{50}$ \\
\hline $111209 \mathrm{~A}$ & $2011 \mathrm{kl}$ & 0.67702 & 10000 & $58.2(7.3)$ & $520(89)$ & $9.76 \times 10^{49}$ \\
\hline $120422 \mathrm{~A}$ & $2012 \mathrm{bz}$ & 0.28253 & 5.4 & $0.024(0.008)$ & $<72$ & $5.70 \times 10^{49}$ \\
\hline 120714B & $2012 \mathrm{eb}$ & 0.3984 & 159 & $0.0594(0.0195)$ & $101.4(155.7)$ & $5.22 \times 10^{48}$ \\
\hline $130215 \mathrm{~A}$ & $2013 \mathrm{ez}$ & 0.597 & 65.7 & $3.1(1.6)$ & $155(63)$ & $7.53 \times 10^{50}$ \\
\hline $130427 \mathrm{~A}$ & 2013cq & 0.3399 & 163 & $81(10)$ & $1028(50)$ & $6.65 \times 10^{51}$ \\
\hline $130702 \mathrm{~A}$ & $2013 \mathrm{dx}$ & 0.145 & 58.881 & $0.064(0.013)$ & $15(5)$ & $1.24 \times 10^{49}$ \\
\hline $130831 \mathrm{~A}$ & 2013fu & 0.479 & 32.5 & $0.46(0.02)$ & $67(4)$ & $2.09 \times 10^{50}$ \\
\hline $171205 \mathrm{~A}$ & 2017iuk & 0.0368 & $190.5(33.9)$ & $0.0022(0.0006)$ & $125(141)$ & $3.16 \times 10^{47}$ \\
\hline
\end{tabular}

$4 \mathrm{~K} \times 4 \mathrm{~K}$ CCD Imager, the ARIES Devasthal Faint Object Spectrograph and Camera (ADFOSC, Omar et al. 2019), High-resolution spectrograph (Pandey et al. 2019), Multi-integral field unit optical spectrograph (DOTIFS, Chung et al. 2014), TIFR-ARIES near-infrared spectrometer (TANSPEC), and TIFR near-infrared imaging camera (TIRCAM2, Baug et al. 2018) are other back-end instruments to be used for the spectroscopy with the $3.6 \mathrm{~m}$ DOT.

GRBs are the highly energetic cosmic stellar explosions observed in the known Universe. These are the energetic jetted explosions having non-thermal spectrum and observed uniformly (isotropic distribution) all over the sky (Fishman \& Meegan 1995; Kumar \& Zhang 2015). GRBs are mainly observed from $\sim 10 \mathrm{keV}-10 \mathrm{GeV}$, with isotropic energy of $10^{50}$ to $10^{55} \mathrm{erg}$ and mostly sustained in a time range of $10^{-3}$ to $10^{3}$ seconds. Due to the high luminosity of GRBs, they even can be tracked down at very high redshifts $(z>8-10)$, making them potential candidates for augmenting cosmological studies and high energy astrophysical mechanisms. Based on the time duration and hardness ratios of the GRBs, they can be distinguished into two categories: Short GRBs (SGRBs) and Long GRBs (L-GRBs). GRBs with duration smaller than 2 seconds are called S-GRBs and those with duration longer than 2 seconds are termed as L-GRBs (Zhang \& Meszaros 2004). Short- and Long-GRBs are suggested to originate from two different types of progenitors: binary mergers and core- collapse of massive stars, respectively (Piran 1999; Zhang 2011).

In the past 2 decades, the most interesting aspects of this field are (i) Correlation of some of the L-GRBs with H-stripped core-collapse supernovae (CCSNe) (ii) Fermi-LAT observed GeV emission in the case of some of the L-GRBs and their correlation with lower-energy X-ray/Gamma-ray emissions. For more than a dozen of L-GRBs, the signatures of $\mathrm{H}$ stripped CCSNe have been detected as a red bump in the late-time afterglow light-curve (Kumar \& Zhang 2015). In 1998, BeppoSax and BATSE detected a faint L-GRB 980425 of duration about 35 seconds, and just after 60 hours, they observed a bright SN (SN $1998 \mathrm{bw}$ ) at the same location (Castro-Tirado et al. 1998; Galama et al. 1998).

From demographic studies of the relative rates of GRBs and CCSNe, it is evident that only a tiny fraction $(\sim 1 \%)$ of core-collapse events can produce Type Ibc SNe with detectable GRBs (Bloom et al. 1998; Berger et al. 2011). Even, Ic-Broad Lined (Ic$\mathrm{BL}) \mathrm{SNe}$, which themselves comprise a small fraction of the CCSNe rate, are seldom associated with LGRBs (Guetta \& Della 2007).

After the discovery of $\mathrm{H}$-stripped superluminous SNe (SLSNe I, Quimby et al. 2011; Gal-Yam 2012), it was speculated that they could also be associated with L-GRBs (Greiner et al. 2015). SLSNe I are very rare, $\sim 10000$ times rare but $\sim 100$ times brighter than normal H-stripped CCSNe (Neill et al. 2011; 
Quimby et al. 2013). SLSNe I are mainly found in low mass dwarf galaxies, whereas, some of the LGRBs are also found in dwarf galaxies, mainly that have low metallicities (Conselice et al. 2005; Angus et al. 2016; Schulze et al. 2018). SLSNe I have perhaps similar kind of central engines to those of L-GRB, however, the former does not dump all the energy in jets and diffuse energy slowly after a few expansion time scale (Cano et al. 2017). Recently, connections observed between L-GRB 111209A and SLSN $2011 \mathrm{kl}$ has opened a new window to explore whether SLSNe I are also associated with L-GRBs (Greiner et al. 2015; Kann et al. 2016, 2019). To understand this new aspect, the Blue supergiant model was suggested for the L-GRBs and SLSNe connection by Nakauchi et al. (2013). Misaligned magnetar model for magnetar formalization and jet formation was suggested by Margalit et al. (2018) to explain possible connections between L-GRBs and SLSNe. In some of the recent works, it has been attempted to probe similarity between GRBs and SLSNe I on the basis of spectral similarity of their host galaxies (Castro-Tirado et al. 2001; Lunnan et al. 2014; Contini 2018). Zou \& Cheng (2018) tried to unify the model which can describe all the events (normal SN-Ic, SN-Ic+GRB, $\mathrm{SN}-\mathrm{Ic}+\mathrm{XRF}, \mathrm{SN}-\mathrm{Ic}-\mathrm{BL}$, and SLSNe) in a single picture on the basis of formation and penetration of jets. The well-observed L-GRBs those were found to be connected with CCSNe including GRB 171205A (D'Elia et al. 2018; Wang et al. 2018; Suzuki et al. 2019) are listed in Table 1 (Cano et al. 2017, and references therein).

In this study, we will discuss the importance of $3.6 \mathrm{~m}$ DOT to understand the time-critical events in Section 2. Section 3 discusses the first-light instrument called $4 \mathrm{~K} \times 4 \mathrm{~K}$ CCD optical Imager. Some of the science goals covered by the instrument are also discussed, e.g., Supernovae, GRBs, and possible connections among the same. Optical photometric unpublished Bessell $R$-band data of L-GRB171205A is also mentioned in Section 4, which was obtained using $4 \mathrm{~K} \times 4 \mathrm{~K}$ CCD Imager at $3.6 \mathrm{~m}$ DOT. We summarize results in Section 5.

\section{IMPORTANCE OF THE 3.6M DOT FOR TIME DOMAIN ASTRONOMY}

The $3.6 \mathrm{~m}$ DOT situated in the middle of the 180 wide belts between Canary Islands $(20 \mathrm{~W})$ and Eastern Australia (160 E) and is very important to do the optical photometry and the spectroscopy of timecritical events. To investigate GRBs afterglow, CC$\mathrm{SNe}$, and the connections among these are some of the interesting goals that could be attempted with the help of the $3.6 \mathrm{~m}$ DOT and the back-end instruments (Pandey 2016). Because of the bigger aperture of $3.6 \mathrm{~m}$ DOT, observations of the fainter host galaxy of GRBs and SLSNe I $\left(\mathrm{M}_{B} \sim-17.3 \mathrm{mag}\right)$ can be obtained, which could probe the new aspects of LGRBs-SLSNe I connections and possible similarity in their environment. Optical afterglow of short-duration GRBs, newly discovered various types of $\mathrm{SNe}$, and other interesting energetic transients are also aimed to be monitored with the $3.6 \mathrm{~m}$ DOT (Pandey 2013, 2016). Explosive transients discovered within the other multi-wavelength facilities like ASTROSAT (the first Indian multi-wavelength satellite), GMRT, and other survey robotic telescopes are also planned to be observed using this facility and other complementary observational facilities at ARIES.

There is a range of time scale variability (from seconds to years) among different astronomical transients. The variability may be intrinsic or the extrinsic property of the object, e.g., Active galactic nuclei or Asteroids, respectively. There are different types of explosions exhibiting a diminishing light curve with time (SNe, GRBs, AGN flares, etc.). Nowadays, we are lucky enough to have the technology to detect such transients with a time scale of seconds after the burst, thanks to the robotic survey telescopes. Transients can emerge anytime in any direction of the sky, so such events are considered for observations under the globally recognized Target of Opportunity ( $\mathrm{ToO}$ ) program. We have to respond pretty fast to observe these transients because in a very short time scale they fall below the detection limit. Usually, the afterglow of GRBs are observed at $\sim 16-18 \mathrm{mag}$ and turns fainter within the time scale of hours to days (Kann et al. 2011). In the few cases, the afterglows are very faint, i.e., $\sim 23$ mag or even fainter, which demands larger telescopes for detection and follow-up observations. Optical photometric studies of the afterglow of GRBs and their host galaxies along with the measurements at other wavelengths are necessary to know more about the surrounding of the progenitors, nature of the dark GRBs, and their physical mechanisms (Kumar \& Zhang 2015). Correlation of some of the nearby GRBs with SNe could also be probed using photometric observations at late times. To trace the evolution of such transients, time-critical observations are required and undoubtedly the $3.6 \mathrm{~m}$ DOT is very crucial for such observations. 

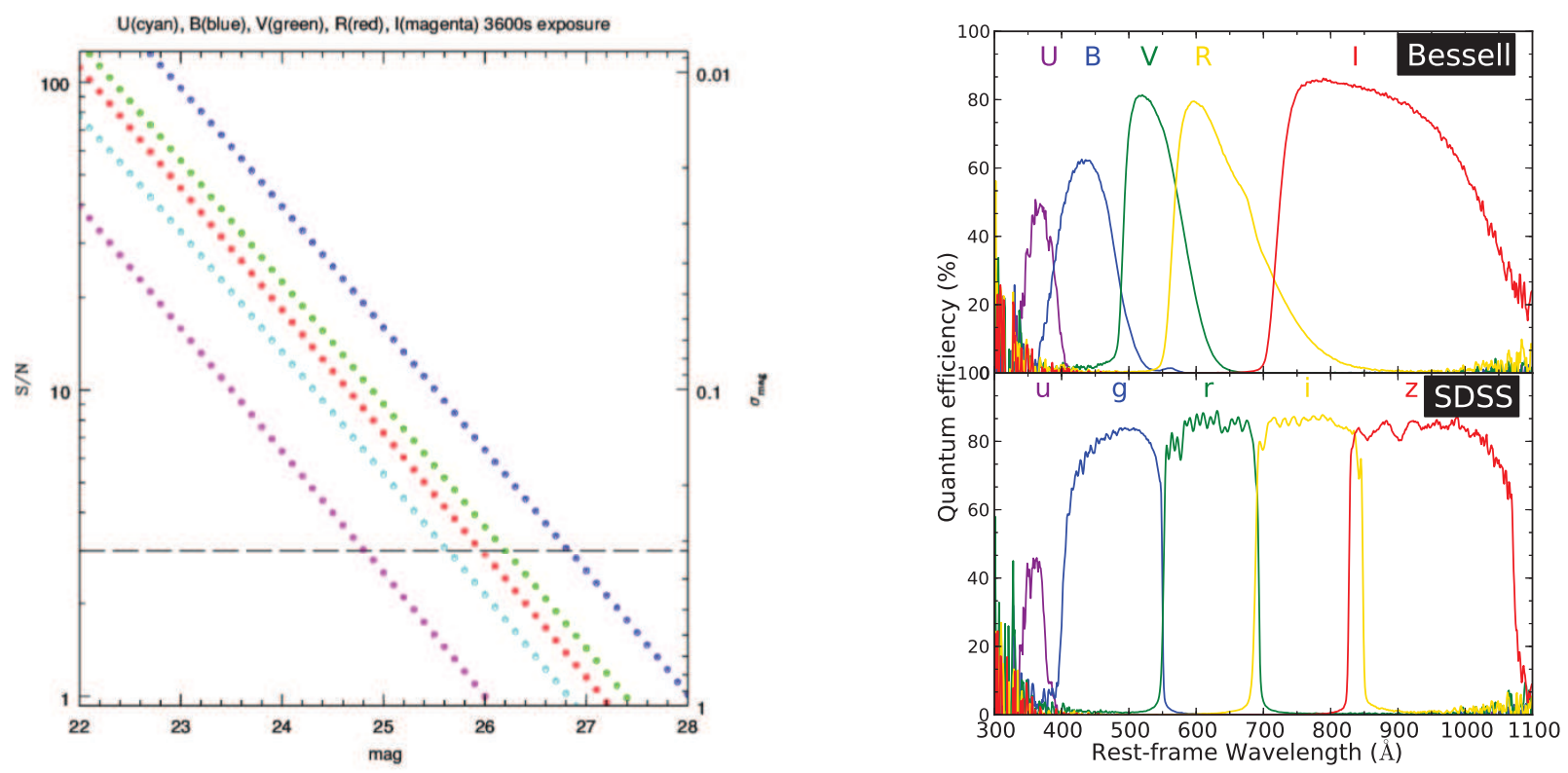

Fig. 1. Left: A simulated plot of magnitude versus signal-to-noise ratio $(\mathrm{S} / \mathrm{N})$ and corresponding error in the magnitude determinations of the $3.6 \mathrm{~m}$ DOT with the proposed $15 \mu \mathrm{m} 4 \mathrm{~K} \times 4 \mathrm{~K}$ CCD camera. This panel is taken from Pandey et al. (2018). Right: Quantum efficiency curves of the Bessell and SDSS set of filters used with the $4 \mathrm{k} \times 4 \mathrm{k}$ CCD Imager.

\section{3. $4 \mathrm{~K} \times 4 \mathrm{~K}$ CCD IMAGER: A FIRST-LIGHT BACK-END INSTRUMENT}

The ARIES STA4150 CCD camera is a highperformance astronomical imaging system developed by Semiconductor Technology Associates, Inc.(STA). The camera is based on a $4 \mathrm{~K} \times 4 \mathrm{~K} 15 \mu \mathrm{m}$ backside-illuminated STA4150 CCD and STA Archon controller. $4 \mathrm{~K} \times 4 \mathrm{~K}$ CCD Imager $^{6}$, with motorized filter-housing, is the first light instrument mounted at the axial port of the $3.6 \mathrm{~m}$ DOT. It has a blue-enhanced back-illuminated CCD chip. Five nominal gain settings $\left(1,2,3,5\right.$, and $\left.10 \mathrm{e}^{-} / \mathrm{ADU}\right)$ with three readout speeds $(100 \mathrm{kHz}, 500 \mathrm{kHz}$, and 1 $\mathrm{MHz}$ ) are there to choose. The detailed study along with the schematic diagram of $4 \mathrm{~K} \times 4 \mathrm{~K}$ CCD Imager is presented in Pandey et al. (2018). Liquid Nitrogen $\left(\mathrm{LN}_{2}\right)$ in CCD Dewar is filled at pressure $<5$ milli Torr. The CCD is cooled up to $-120^{\circ} \mathrm{C}$ and is maintained at it, which helps in using it for even longer exposures to detect fainter objects. $\mathrm{LN}_{2}$ holding time is estimated to be around 13 to 14 hours. The CCD has three integration modes: Non-Multi Pinned Phase (Non-MPP), MPP and, Clocked Antiblooming $(\mathrm{CAB})$, anyone can be chosen at a time. The Non-MPP has a higher dark current but somewhat higher full well capacity. On the other hand, MPP mode has the lowest dark current, whereas, $\mathrm{CAB}$ suppresses blooming by clocking the image

\footnotetext{
${ }^{6}$ http://www.sta-inc.net
}

area during integration but has a higher dark current. Based on performance, Non-MPP is the desired mode of operation (Pandey et al. 2018). Desired integration time can be set in milliseconds but time less than 10 milliseconds may have uncertainty due to mechanical shutter motion. A bonn shutter of $125 \mathrm{~mm}$ is used in the $4 \mathrm{~K} \times 4 \mathrm{~K}$ CCD Imager with a mechanism to run the drivers of the shutter assembly through the CCD controller software. The CCD has 16-bit ADC thus it can represent 65535 ADU counts. To attempt various other scientific goals, $\sim 6.5^{\prime} \times$ $6.5^{\prime}$ central field of view of the telescope along with the Imager and filter wheel can be obtained. The left panel of Figure 1 presents the quantum efficiency curve as a function of wavelength, taken from Pandey (2016); Pandey et al. (2018).

Both, the software and hardware parts of the motorized filter wheels of the CCD Imager were designed, developed, and implemented in ARIES. There are two filter wheels, the first filter wheel has a set of six Bessell's filters $(U, B, V, R, I$, and $C$ ), whereas the second one has a set of six SDSS's filters $(u, g, r, i, z$, and $c)$. The quantum efficiency curves with respect to the rest-frame wavelengths for all Bessell and the SDSS filters are shown in the right panel of Figure 1. In the Imager, Micro-controller and the GUI software are used to set the positions of all the discussed filters. The control unit consists of a PIC micro-controller having Serial Communications Interface (SCI) module USART (Univer- 

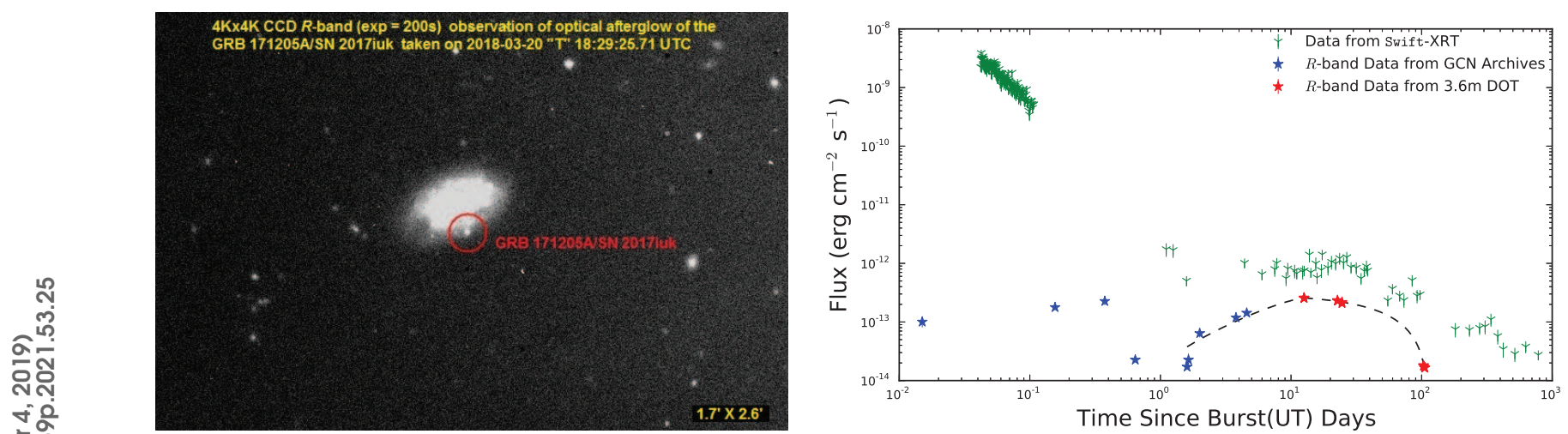

Fig. 2. Left: Identification chart of GRB 171205A/SN 2017iuk. The $R$-band image obtained using the $3.6 \mathrm{~m}$ DOT on 2018 March 20 is shown. The field of view is $\sim 1.7^{\prime} \times 2.6^{\prime}$ and the GRB 171205A/SN 2017iuk is shown with the red circle. Right: The temporal evolution of GRB 171205A is shown. Red data points are obtained using the 3.6m DOT and blue $R$-band data points are taken from GCNs. Temporal evolution observed by the Swift-XRT is also plotted with green points. An SN bump is observed, which seems to have peak at $\sim 15$ days since burst.

sal Synchronous Asynchronous Receiver Transmitter) and a circuit that converts the signal levels from RS-232 compatible with the USART's logic levels and vice-versa. Homing is achieved after powering ON, using the Hall Effect sensors. A detailed log of commands, status, and errors are continuously generated by the GUI interface. Software and control units are successfully tested and integrated with the CCD Imager. Deeper imaging of Galactic and extragalactic point sources $(B \sim 27 \mathrm{mag})$ and objects with low surface brightness could be performed using the $4 \mathrm{~K} \times 4 \mathrm{~K}$ CCD Imager in several broad-band filters (Bessell UBVRI and SDSS ugriz). On $30^{\text {th }}$ March 2016, prime ministers of India and Belgium technically and officially activated the $3.6 \mathrm{~m}$ DOT along with $4 \mathrm{~K} \times 4 \mathrm{~K}$ CCD Imager mounted at the axial port of the telescope. Deep imaging of the star clusters, GRB afterglows, SNe, host galaxy of the LGRBs and SLSNe I, optical variability of AGNs, EUV-bright and soft X-ray sources and optical follow-up of interesting sources from 4m-ILMT, ASTROSAT, GMRT, and other robotic survey telescopes are some of the major goals to be covered using the $4 \mathrm{~K} \times 4 \mathrm{~K}$ CCD Imager (Pandey 2016; Pandey et al. 2018).

\section{PHOTOMETRIC STUDY OF GRB 171205A/SN 2017IUK}

To explore GRB-SN connections, the study of GRB 171205A/SN 2017iuk is performed using the $R$-band data obtained from $3.6 \mathrm{~m}$ DOT with the $4 \mathrm{~K} \times 4 \mathrm{~K}$ CCD Imager, see Left panel of Figure 2 . GRB 171205A/SN 2017iuk is the third nearest GRB$\mathrm{SN}$ ever known and located at $\mathrm{z}=0.0368$. The Swift detected L-GRB 171205A presents lowermost blackbody temperature in comparison to other well stud- ied L-GRBs and possibly associated with a broadlined SN 2017iuk (D'Elia et al. 2018; Wang et al. 2018; Suzuki et al. 2019). For GRB 171205A, an SN bump (at $\sim 13$ days after the burst) in UVOT $u, b$, and $v$ bands are observed by D'Elia et al. (2018). Whereas, Wang et al. (2018) also confirmed the association of a broad-lined SN connection with GRB 171205A, which seems to have a possible CSM interaction powering mechanism (Suzuki et al. 2019).

For the optical photometric study of GRB $171205 \mathrm{~A} / \mathrm{SN}$ 2017iuk, we obtained the Bessell $R$ band data using $3.6 \mathrm{~m}$ DOT and the back-end instrument $4 \mathrm{k} \times 4 \mathrm{k}$ Imager. During the observations, gain and the readout noise values had been fixed to $5 \mathrm{e}^{-} / \mathrm{ADU}$ and $10 \mathrm{e}^{-}$, respectively. Images were taken in the Bessell $R$-band in good weather conditions (air-mass $<1.4$ ) with an exposure time of 200 to 300 seconds. The photometric $R$-band observation of GRB 171204A/SN 2017iuk with $3.6 \mathrm{~m}$ DOT started from $\sim 12$ to 105 days after the burst. Along with the GRB field, bias frames for removing zero integration noise, and flat frames to remove non-uniformity of pixel to pixel response were also taken. The pre-processing of the data such as bias subtraction, flat fielding, and high energy particles such as cosmic rays were removed by using the packages available in $I R A F^{7}$. To estimate the instrumental magnitudes, we performed PSF photometry using packages in the DAOPHOT-II. To convert the instrumental magnitudes into standard magnitudes, we used magnitudes of the local standard stars.

\footnotetext{
${ }^{7}$ IRAF is distributed by the National Optical Astronomy Observatories, which are operated by the Association of Universities for Research in Astronomy, Inc., under a cooperative agreement with the National Science Foundation.
} 
In our set of observations with the $3.6 \mathrm{~m}$ DOT, we have very few data points for this GRB. So, to trace the full $R$-band evolution of the GRB $171205 \mathrm{~A}$, we took several data points at different epochs from GCN 22182, GCN 22186, GCN 22188, GCN 22193, GCN 22195, GCN 22197, GCN 22198, GCN 22205, GCN 22223 and GCN 22224. SDSS $r$ and $i$-band data taken from GCNs were converted to Bessell $R$ band data using the transformation equations given by Jordi et al. (2006). The galactic reddening corrected magnitudes were converted to monochromatic fluxes using the zero points from Bessell et al. (1998).

The analyzed photometric $R$-band light curve of GRB 171205A is plotted with stars, see Figure 2. The light curve of GRB 171205A shows a segmented power-law decay, whereas the late-time light curve shows a clear SN bump. Association of SN (SN 2017iuk) with GRB 171205A can be traced clearly from our late-time light curve bump. As we can see, after $\sim 3$ days since burst, the SN flux start dominates and shows a clear bump in the light curve (fitted with high order spline function). The SN bump seems to peak at $\sim 15$ days since burst, which is close to the value observed by D'Elia et al. (2018). Similar bump at nearly same epoch is also shown by Swift-XRT data of the GRB 171205A, shown with the green points in Figure 2. So, $R$-band light curve clearly indicates the SN association with GRB 171205A. For a significant comparison, properties of the GRB 171205A taken from D'Elia et al. (2018); Wang et al. (2018) is tabulated in Table 1 along with the other well-observed GRBs-SNe that are taken from Cano et al. (2017, and references therein), a detailed analysis of such observed properties would be published in Kumar et al. (2020).

\section{SUMMARY}

Longitudinal advantage of the $3.6 \mathrm{~m}$ DOT makes it very crucial for time-domain astronomy. Using $4 \mathrm{~K} \times 4 \mathrm{~K} \mathrm{CCD}$ Imager and Test-WFS, the performance of the $3.6 \mathrm{~m}$ DOT has been tested. The $3.6 \mathrm{~m}$ DOT along with the blue-enhanced $4 \mathrm{~K} \times 4 \mathrm{~K}$ CCD Imager will prove to be very crucial in observing transients up to a very fainter limit $(\sim 25 \mathrm{mag})$ in Bessell and SDSS broad-band filters. This will definitely help astronomers to observe and trace the evolution of the new transients, which may resolve many unanswered questions regarding progenitors, environment, and physical mechanisms of time-critical events. Deep imaging using the Imager will also help to understand the late-time optical light curve evolution of SNe and GRBs, whereas, observation of the host galaxies (faint and low-mass dwarf galaxies with lower metallicity) of L-GRBs and SLSNe I could probe the possible connections among these energetic events. It may also reveal some useful and concrete insights on the possibilities of the powering mechanism of SLSNe. Optical photometric follow up of the objects detected by the 4m-ILMT, ASTROSAT, and GMRT will also be one of the important goals of the $4 \mathrm{~K} \times 4 \mathrm{~K}$ CCD Imager.

Based on the photometric Swift-UVOT and XRT observations of GRB 171205A along with the spectroscopic study of SN 2017iuk, previous studies claimed it as an event showing GRB-SN connection. So, we have also presented the photometric $R$-band study of this close-by burst $(\mathrm{z}=0.0368)$. GRB $171205 \mathrm{~A}$ is one of the densely covered and wellstudied events which has an association of broadlined SN-Ic. This study aims to add an important optical counterpart of GRB 171205A in the literature. Our GRB 171205A observations in Bessell $R$-band using $3.6 \mathrm{~m}$ DOT along with some archival data in the same band also confirms the late-time SN bump in the light curve of GRB 171205A. The $R$-band light curve of GRB 171205A seems to have a clear SN bump after $\sim 3$ days since burst, which seems to peak at $\sim 15$ days. This late time bump in the optical afterglow of GRB 171205A confirms the association of SN with this event.

Acknowledgments: A.K. is also grateful to the staff members of the $3.6 \mathrm{~m}$ DOT for their consistent support and help. This work has utilized the NED, which is operated by the Jet Propulsion Laboratory, California Institute of Technology, under contract with NASA. We acknowledge the availability of NASA ADS services.

\section{REFERENCES}

Angus, C. R., Levan, A. J., Perley, D. A., et al. 2016, MNRAS, 458, 84

Baug, T., Ojha, D. K., Ghosh, S. K., et al. 2018, JAI, $750003 \mathrm{~B}$

Berger, E., Chornock, R., Holmes, T. R., et al. 2011, ApJ, 743, 204

Bessell, M. S., Castelli, F., \& Plez, B. 1998, A\&A, 333, 231

Bloom, J. S., Kulkarni, S. R., Harrison, F., et al. 1998, ApJ, 506, 105

Cano, Z., Wang, S. Q., Dai, Z. G., et al. 2017, AdAst, 2017,5

Castro-Tirado, A. J., Gorosabel, J., Costa, E., et al. 1998, AIPC, 428, 489

Castro-Tirado, A. J., Sokolov, V. V., Gorosabel, J., et al., 2001, A\&A, 370, 398

Chung, H., Ramaprakash, A. N., Omar, A., et al. 2014, SPIE, 9147E, 0VC

Conselice, C. J., Vreeswijk, P. M., Fruchter, A. S., et al. 2005, ApJ, 633, 29 
Contini, M. 2018, ArXiv e-prints, arXiv:1801.03312

D'Elia, V., Campana, S., D'Ai, A., et al. 2018, A\&A, 619, 66

Fishman G. J. \& Meegan C. A. 1995, ARA\&A, 33, 415

Galama, T. J., Vreeswijk, P. M., van Paradijs, J., et al. 1998, Natur, 395, 670

Gal-Yam, A. 2012, Sci, 337, 927

Greiner, J., Mazzali, P. A., Kann, D. A., et al. 2015, Natur, 523, 189

Guetta, D. \& Della V. M. 2007, ApJ, 657, 73

Jordi, K., Grebel, E. K., \& Ammon, K. 2006, A\&A, 460, 339

Kann D. A., Klose S., Zhang B., et al. 2011, ApJ, 734, 96

Kann, D. A., Schady, P., Olivares E., F., et al. 2016, arXiv:1606.06791

Kann, D. A., Schady, P., Olivares E., F., et al. 2019, A\&A, 624, 143

Kumar, P. \& Zhang, B. 2015, PhR, 561, 1

Kumar, B. 2015, ASInC, 12, 73

Kumar, A., et al. 2020, under preparation

Lunnan, R., Chornock, R., Berger, E., et al. 2014, ApJ, 787,138

Leloudas, G., Schulze, S., Krühler, T., et al. 2015, MNRAS, 449, 917

Margalit, B., Metzger, B. D., Berger, E., et al. 2018, MNRAS, 481, 2407
Nakauchi, D., Kashiyama, K., Suwa, Y., et al. 2013, ApJ, 778,67

Neill, J. D., Sullivan, M., Gal-Yam, A., et al. 2011, ApJ, 727,15

Omar, A., Kumar, T. S., Krishna Reddy, B., et al. 2019, arXiv:1902.05857

Pandey, S. B. 2016, RMxAC, 48, 83

Pandey, J. C., Pant, J., \& Joshi, Y. C. 2019, BSRSL, 88, 43

Pandey S. B. 2013, JApA, 34, 157

Pandey S. B., Yadav R. K. S., Nanjappa, N., et al. 2018, BSRSL, 87, 42

Piran T. 1999, PhR, 314, 575

Quimby, R. M., Kulkarni, S. R., Kasliwal, M. M., et al. 2011, Natur, 474, 487

Quimby, R. M., Yuan, F., Akerlof, C., \& Wheeler, J. C. 2013, MNRAS, 431, 912

Sagar, R. 2000, Current Science, 78, 1076

Schulze, S., Krühler, T., Leloudas, G., et al. 2018, MNRAS, 473, 1258

Suzuki, A., Maeda, K., \& Shigeyama, T. 2019, ApJ, 870, 38

Wang, J., Zhu, Z. P., Xu, D., et al. 2018, ApJ, 867, 147

Zou, Y. C. \& Cheng, K. S. 2018, PASA, 35, 32

Zhang, B. \& Meszaros, P. 2004, IJMPA, 19, 2385

Zhang, B. 2011, CRPhy, 12, 206 